\title{
Asphalt Mixture for the First Asphalt Concrete Directly Fastened Track in Korea
}

\author{
Seong-Hyeok Lee, ${ }^{1}$ Dae-Wook Park, ${ }^{2}$ Hai Viet Vo, ${ }^{2}$ and Samer Dessouky ${ }^{3}$ \\ ${ }^{1}$ Korea Railway Research Institute, 176 Cheldo Bangmulgwan-ro, Uiwang, Gyeonggi-do 437-757, Republic of Korea \\ ${ }^{2}$ Department of Civil Engineering, Kunsan National University, 558 Daehak-ro, Kunsan, Chellabuk-do 573-701, Republic of Korea \\ ${ }^{3}$ Department of Civil and Environmental Engineering, University of Texas, San Antonio, TX 78249, USA \\ Correspondence should be addressed to Dae-Wook Park; parkdw@hotmail.com
}

Received 5 March 2015; Accepted 18 March 2015

Academic Editor: João M. P. Q. Delgado

Copyright (C) 2015 Seong-Hyeok Lee et al. This is an open access article distributed under the Creative Commons Attribution License, which permits unrestricted use, distribution, and reproduction in any medium, provided the original work is properly cited.

\begin{abstract}
The research has been initiated to develop the asphalt mixtures which are suitable for the surface of asphalt concrete directly fastened track (ADFT) system and evaluate the performance of the asphalt mixture. Three aggregate gradations which are upper (finer), medium, and below (coarser). The nominal maximum aggregate size of asphalt mixture was $10 \mathrm{~mm}$. Asphalt mixture design was conducted at 3 percent air voids using Marshall mix design method. To make impermeable asphalt mixture surface, the laboratory permeability test was conducted for asphalt mixtures of three different aggregate gradations using asphalt mixture permeability tester. Moisture susceptibility test was conducted based on AASHTO T 283. The stripping percentage of asphalt mixtures was measured using a digital camera and analyzed based on image analysis techniques. Based on the limited research results, the finer aggregate gradation is the most suitable for asphalt mixture for ADFT system with the high TSR value and the low stripping percentage and permeable coefficient. Flow number and beam fatigue tests for finer aggregate asphalt mixture were conducted to characterize the performance of asphalt mixtures containing two modified asphalt binders: STE-10 which is styrene-butadienestyrene (SBS) polymer and ARMA which is Crum rubber modified asphalt. The performance tests indicate that the STE-10 shows the higher rutting life and fatigue life.
\end{abstract}

\section{Introduction}

Hot mix asphalt (HMA) was first developed for applying to the track structure to partly replace the conventional granular material and now is selectively considered as an option for new mainline tracks, yards, and terminal construction due to lower delivering and placing cost compared to conventional granular subballast [1]. Hensley and Rose [2] found that HMA mat is capable of performing as an elastic layer under the railway instead of open-graded unbound ballast layer. HMA is suitable for railway substructure to enhance performance, support dynamic, and vibrated loading especially at the effective depth of 0-2 meters [3]. Viscoelastic strength and modulus of HMA can make it more sustainable for using as high-speed railway substructures [4] and also no damage or crack of the asphalt is detected after many years of heavy traffic under various conditions [5]. Fang et al. [6] concluded that air voids content and permeability of asphalt mixture is strongly affected by the aggregate gradation but not correlated with normal maximum aggregate size. Rose et al. [7] suggested that the loading conditions in trackbeds are different from those in highway pavements, so the asphalt content of HMA trackbeds should be $0.5 \%$ higher than that considered optimum for highway applications with air voids of 1 to $3 \%$ to facilitate adequate strength and an impermeable mat. Sangsefidi et al. [8] postulated to adopt the optimum mixture; the aggregate gradation is considered as an important characteristic, since it affects the rutting resistance and the moisture susceptibility of the asphalt mixtures. Merusi et al. [9] applied a digital image analysis method to identify the stripping surface to quantitatively evaluate the stripping resistant of asphalt mixture. It offers a good observation and qualification of stripping. The impermeable asphalt layer can prevent stripping and possible contamination of the lower layer by vertical hydraulic transport of mud and fines [10]. 


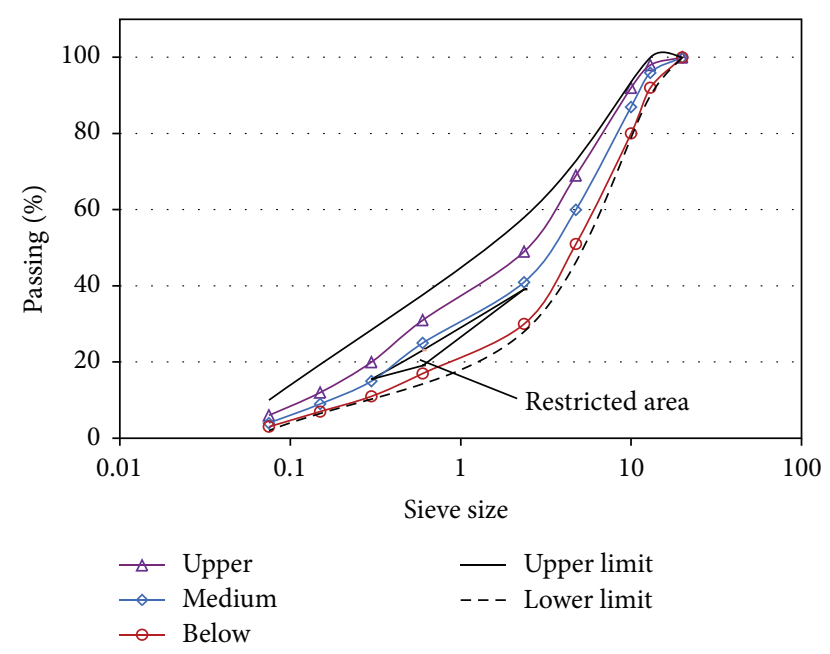

FIGURE 1: Aggregate gradations in 0.45 power.

The purposes of this study are to evaluate the asphalt mixtures with three aggregate gradations, upper, medium, and below, to evaluate which are suitable for the surface of Asogakt Concrete Directly Fastened (ADFT) system. The Marshall mix design method was conducted to determine the optimum asphalt binder content for asphalt mixes with different aggregate gradation. Digital images captured from wet indirect tensile (IDT) test were analyzed and compared with its TSR obtained from dry and wet conditioned specimens in AASHTO T 283 test. The falling head permeameter was used to determine the permeability of the asphalt mixtures in the laboratory in order to find the impermeable mixtures correlated with the aggregate gradations. The performance of asphalt concrete mixtures which has upper gradation was investigated using flow number and beam fatigue test using two types of modified asphalt binders.

\section{Materials and Mix Design}

2.1. Materials. Asphalt binder STE-10 which is styrene-butadiene-styrene (SBS) polymer was used for evaluating aggregate gradations. The aggregates with three gradations, upper (finer), medium, and below (coarser), have bulk specific gravities, $G_{\mathrm{sb}}$, of $2.563,2.578$, and $2.594 \mathrm{~g} / \mathrm{cm}^{3}$, respectively, determined according to AASHTO T 85 [11] and AASHTO T 84 [12] tests. The aggregate gradations were chosen between the upper and lower control limits without passing through the restricted area based on Superpave mix design method with aggregate maximum size of $12.5 \mathrm{~mm}$ [4]. The details of aggregate gradations are shown in Figure 1.

2.2. Mix Design. Asphalt mix design is conducted based on Marshall mix design method [4], and samples of asphalt mixtures were prepared with three replicates at three different asphalt contents. The optimum asphalt contents selected at an air void of $3 \%$ were $5.5 \%, 5.2 \%$, and $5.3 \%$ for upper, medium, and below mixes, respectively. As shown in Table 1, asphalt mixture with below gradation did not meet the flow value criteria as 43.1 .
TABLE 1: Marshall test results at the optimum asphalt content.

\begin{tabular}{lcccc}
\hline Marshall properties & Criteria limit & Upper & Medium & Below \\
\hline Optimum AC (\%) & At 3 & $\mathbf{5 . 5}$ & $\mathbf{5 . 2}$ & $\mathbf{5 . 3}$ \\
Marshall stability (kgf) & $>500$ & 2536.7 & 2162.9 & 1776.1 \\
VMA (\%) & $>13$ & 13.6 & 13.0 & 12.9 \\
VFA (\%) & $70-80$ & 79.3 & 78.8 & 77.9 \\
Flow $(0.01 \mathrm{~cm})$ & $20-40$ & 35.8 & 31.8 & 43.1 \\
$G_{\mathrm{mb}}\left(\mathrm{g} / \mathrm{cm}^{3}\right)$ & - & 2.344 & 2.353 & 2.358 \\
\hline
\end{tabular}

\section{Evaluation of Aggregate Gradations}

3.1. Indirect Tensile (IDT) Test. HMA moisture susceptibility is mostly determined based on the AASHTO T 283 [13]. The damage due to moisture is controlled by the specific limits of the tensile strength ratios (TSR). All specimens were compacted by the gyratory compactor and had air voids of $7 \pm 0.5 \%$. Total six specimens were separated into two subsets: one subset for dry IDT test and the other for wet IDT test. The maximum load was recorded from the indirect tensile tester and the corresponding IDT strength was calculated by

$$
S_{t}=\frac{2000 P}{\pi D t},
$$

where $S_{t}$ is the IDT strength, $\mathrm{kPa}, P$ is the maximum load, $t$ is the thickness of specimen, and $D$ is the diameter of specimen. The tensile strength ratio (TSR) is determined by the following equation from the dry and wet IDT test results:

$$
\operatorname{TSR}=\frac{S_{W}}{S_{D}},
$$

where $S_{W}$ is the average wet IDT strength and $S_{D}$ is the average dry IDT strength. Figure 2 indicates that the aggregate gradation affects to some extent the moisture susceptibility of the asphalt mixtures. The mixtures with upper, medium, and below gradations have TSR values of $82.5 \%, 80.2 \%$, and $81.7 \%$, respectively. Accordingly, all the mixtures met the criteria of a minimum TSR value of $80 \%$ [13], and the mixes with upper (finer) gradation showed better TSR than mixes with medium or below (coarser) gradation. Basically, the water penetration depends on the gradation of aggregate: dense or open graded. Since finer materials tend to fill voids and increase the density, therefore its reduction also affected the TSR in coarser graded mixes. Furthermore, due to breaking of coarser aggregate during compaction, uncoated surface absorbs the water more than the other particles and then leads to stripping. There is low amount of fine material in medium and below aggregate gradation. As a result, although the asphalt mixture with medium aggregate gradation has high dry and wet IDT strength values (Figure 2(a)), but, as seen in Figure 2(b), its moisture resistance or TSR value is the lowest as compared to the others.

3.2. Image Analysis. Two-halves of the samples from the wet IDT test were kept for image stripping analysis. The images were captured to the fractured surface by digital camera 


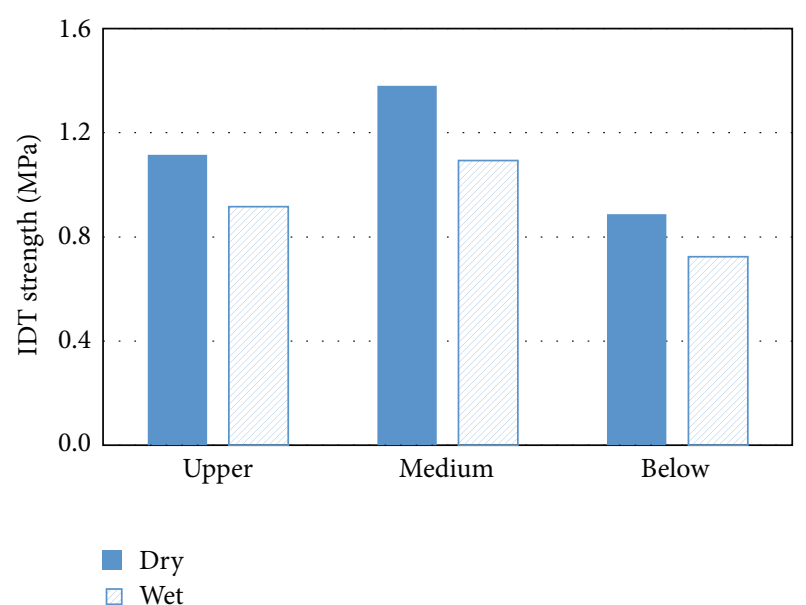

(a) IDT strength

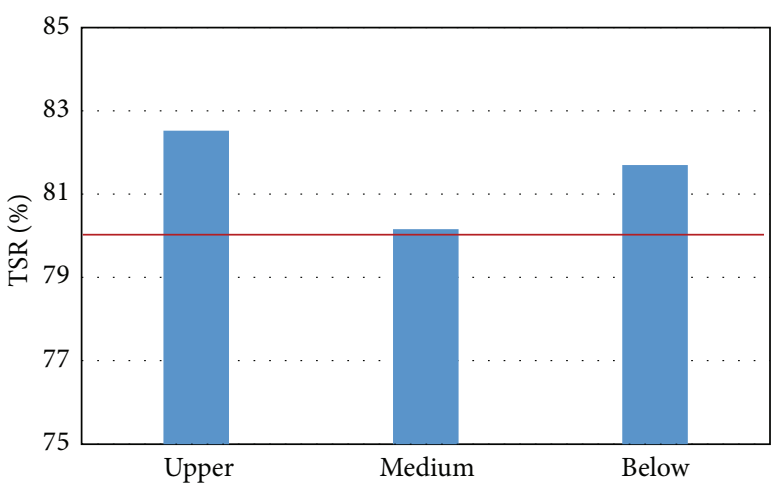

(b) TSR

FIGURE 2: Indirect tensile (IDT) test result.

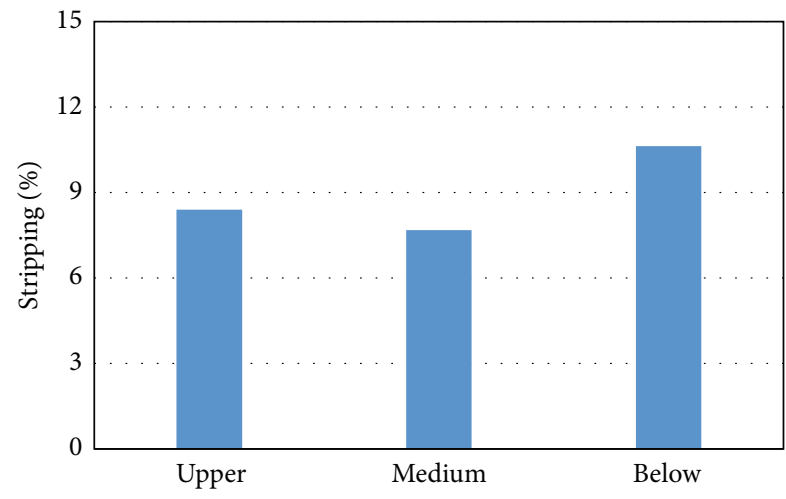

FIGURE 3: Image analysis result.

(SONY DSC-T100 8.1 mega pixels) in an adequate and indirect light condition to prevent creation of light reflection. It is recommended to use a light green color as background during taking picture. With this color, background separation can be done accurately and evenly. Moreover, the angle of images capturing should be perpendicular to the surface of sample. The image analysis program developed using Matlab [14] was employed for determining stripping percentage. The numbers of black and white pixels were counted to determine the stripping area. By determining a threshold value within the grayscale, white and black pixels can be distinguished in binary image. Applying different threshold levels indicated that the global threshold values between 0.16 and 0.17 did not significantly affect the stripping percentage results; however, for an accurate comparison, the analyses of all the images should be done with the same threshold value. The threshold value of 0.165 was chosen to distinguish the white and black pixels. This threshold level seems to be a realistic value to recognize the stripping percentage.

In image analysis test, the lower the stripping percentage is, the less the moisture damage occurs. As seen in Figure 3, the results indicate that the image analysis is basically reliable when its results, stripping percentage, have almost the same trend with the wet IDT values. The IDT test result of the asphalt mixture with medium aggregate gradation is high, and also the image analysis indicates a high stripping resistance (lower stripping percentage), relatively matching the wet IDT strength value. In this study, the stripping percentage values have yet denoted the correlation with TSR values.

3.3. Permeability Test. The permeability of asphalt concrete samples was measured by the permeameter developed by Florida Department of Transportation (FDOT) for testing in the laboratory [15]. The permeable coefficient was computed according to (3). Three replicated tests on the same specimen were performed and averaged:

$$
k=\frac{a L}{A t} \ln \left(\frac{h_{1}}{h_{2}}\right) t_{c},
$$

where $k$ is the permeable coefficient, $a$ is the inside crosssectional area of the graduated cylinder, $L$ is the average thickness of the test specimen, $A$ is the cross-sectional area of the test specimen, $t$ is the elapsed time between $h_{1}$ and $h_{2}$, $h_{1}$ and $h_{2}$ are initial and final head across the test specimen, and $t_{c}$ is the temperature correction for viscosity of water. According to FDOT's criteria for impermeable asphalt mixture, the permeable coefficient measured by the falling head permeameter must not exceed $0.1 \mathrm{~cm} / \mathrm{s}$. The permeability of asphalt concrete is mostly affected by the porosity and air voids distribution. As seen in Figure 4, all mixtures met criteria for impermeable mixture; however, the permeability of the mix with below (coarse) gradation is higher by far than the others. All the air void contents are almost at the same level. Therefore, it is noted that the upper and medium mixes can produce smaller air void pockets than the below.

\section{Performance Evaluation}

According to the previous work [16], the modified asphalt mixtures are suitable for railway substructures compared to 


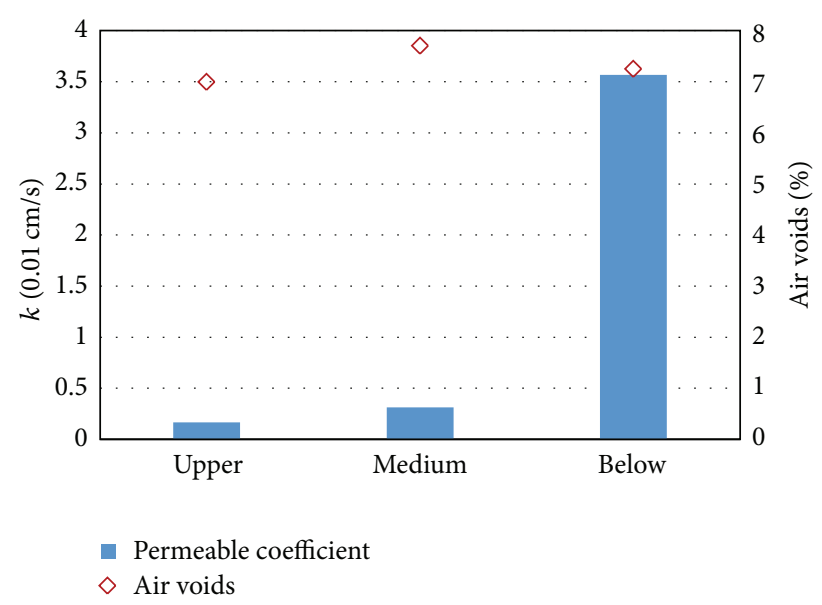

FIgUre 4: Permeability test result.

the original one. For performance evaluation, asphalt mixtures with two types of modified asphalt binder, STE-10 and ARMA, were used for flow number and four point beam fatigue tests.

4.1. Flow Number. The flow number (FN) is a measure of the rutting potential of asphalt mixes [17]. Mixes with high flow number are more resistant to permanent deformation and vice versa. The cylindrical specimen geometry is similar to the dynamic modulus test. Asphalt specimens were subjected to repeated haversine axial cycle with 0.1 and $0.9 \mathrm{sec}$ loading time and rest period, respectively. The test was performed under unconfined conditions at $54^{\circ} \mathrm{C}$ and deviatoric stress of $600 \mathrm{kPa}$. The failure criterion is determined at 15,000 cycles or 50,000 microstrain, whichever occurs first. During FN test, the mix follows three different stages of deformation: primary (Consolidation), steady state, and tertiary [17]. Flow number is the number of load cycles at the minimum rate of permanent axial strain (e.g., constant volume) at which tertiary flow begins. The minimum strain rate was determined at the junction point in between the steady state and tertiary of deformation. The following equation is applied to calculate the strain rate;

$$
\frac{\delta_{s_{i}}}{\delta_{N}}=\frac{\varepsilon_{N_{i+1}}-\varepsilon_{N_{i-1}}}{2 \Delta N},
$$

where $\delta_{s_{i}} / \delta_{N}$ is the strain rate at $N$ th cycle, $\varepsilon_{N_{i+1}}$ is the strain at $(i+1)$ th cycle, $\varepsilon_{N_{i+1}}$ is the strain at $(i-1)$ th cycle, and $\Delta N$ is the number of cycles in between $(i+1)$ th and $(i-1)$ th cycle. In this study, the test was conducted on two replicates with an average FN of 733 and 226 cycles for STE-10 and ARMA mixtures, respectively. Figure 5 represents the strain rate evolution for the two replicates of STE-10 mixtures. Results suggest that the asphalt mixture containing STE-10 exhibits the higher rutting life compared to that of ARMA.

4.2. Four Point Beam Fatigue Test. This test was conducted to determine the fatigue life of asphalt pavement using a $380 \times$ $63 \times 50 \mathrm{~mm}$ beam. Repeated loads were applied until reaching fatigue. Fatigue life is the number of cycles recorded at the failure of asphalt mixtures according to AASHTO T 321 [18].

An in-house steel mold was used to fabricate $405 \times 240 \times$ $75 \mathrm{~mm}$ asphalt concrete slab. The slab is compacted by a steel rod to reach target air voids of 7 to $9 \%$. The slab was sawn approximately $6 \mathrm{~mm}$ from all sides to avoid the air voids clustering at the edge of the slab due to the mold temperature. After that, two standard beams of $380 \times 63 \times 50 \mathrm{~mm}$ were cut from the slab.

The beams were tested by four point loading clamps. The loads on the two inner clamps were cycled with a repeated haversine (sinusoidal) load at frequency of $10 \mathrm{~Hz}$ while the outer ones remained fixed to provide a reaction support, providing a constant bending moment over the center part of the beam. The deflection caused by the loading is measured at the center of the beam. The deformation of the beam centroid was calculated to produce the tensile strain values in the bottom fiber of the beam and collects load and deformation data every 100 cycles.

The test was performed on a strain-controlled condition using a strain level of 650 microstrain at room temperature of $19 \pm 1^{\circ} \mathrm{C}$. The sample was conditioned in a chamber for about two hours at the testing temperature prior to testing. As loading is applied, the beam resilience reduces gradually. The test was terminated when the beam stiffness reduced to $50 \%$ of the initial stiffness, and the number of cycles at that time is referred to as the fatigue life. The flexural stiffness $(S)$ is determined using

$$
S=\frac{\sigma_{t}}{\varepsilon_{t}}
$$

where $\sigma_{t}$ and $\varepsilon_{t}$ are the maximum tensile stress and strain determined as follows:

$$
\begin{gathered}
\sigma_{t}=\frac{0.357 P}{b h^{2}}, \\
\varepsilon_{t}=\frac{12 \delta h}{3 L^{2}+4 a^{2}},
\end{gathered}
$$

where $P$ is the applied load by actuator, $b$ is the average beam width, $h$ is the average beam height, $\delta$ is the maximum deflection at center of beam, $L$ is the average beam length (m), and $a$ is the space between inner clamps $(0.119 \mathrm{~m})$. Examples of the normalized fatigue stiffness reduction with number of cycles for all mixtures are shown in Figure 6. Results suggest that the STE-10 exhibits the higher fatigue life with 50 times greater than the other.

\section{Conclusions}

The ADFT system requires a durable and impermeable asphalt layer under the sleepers. Three types of mixes made with combination of three different aggregate gradations were designed and evaluated for their moisture susceptibility and permeability. The mixtures were designed by Marshall mix design method and tested with IDT test, image analysis 


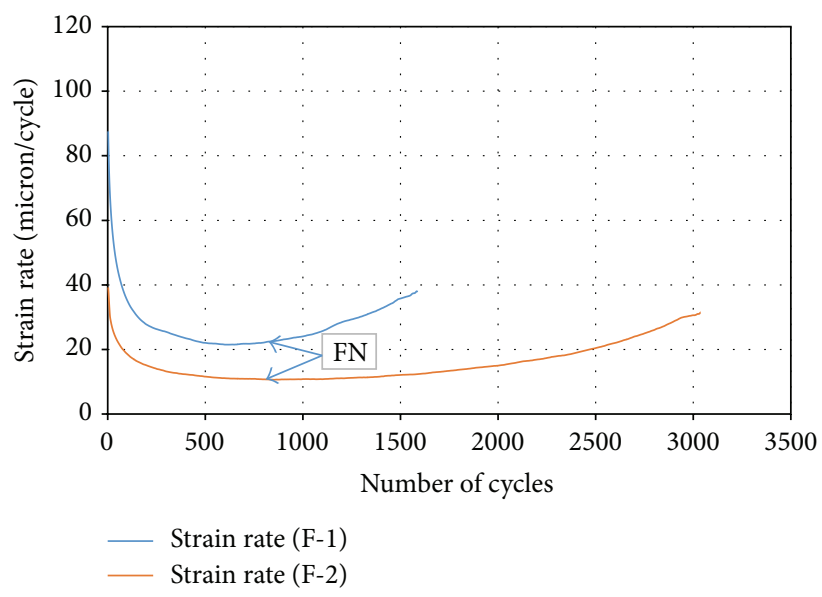

(a) STE-10 replicates

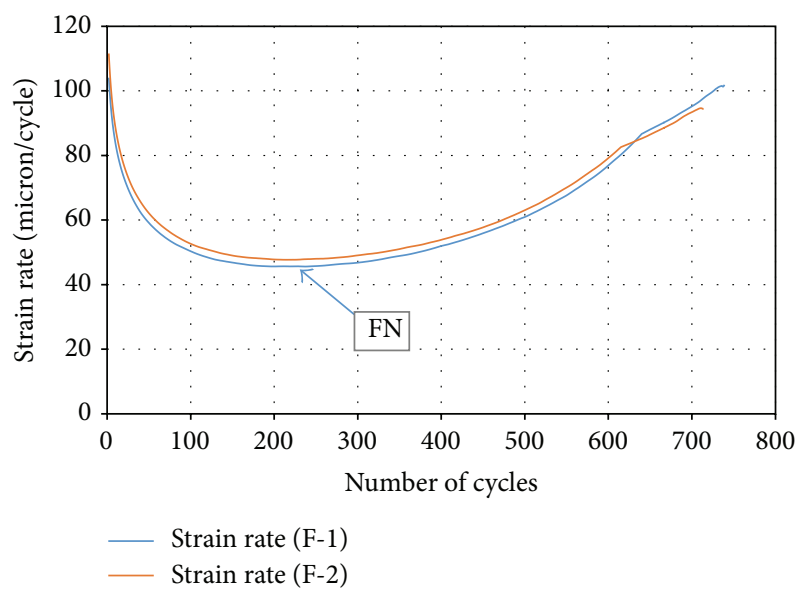

(b) ARMA replicates

FIGURE 5: Permanent strain rate curves.

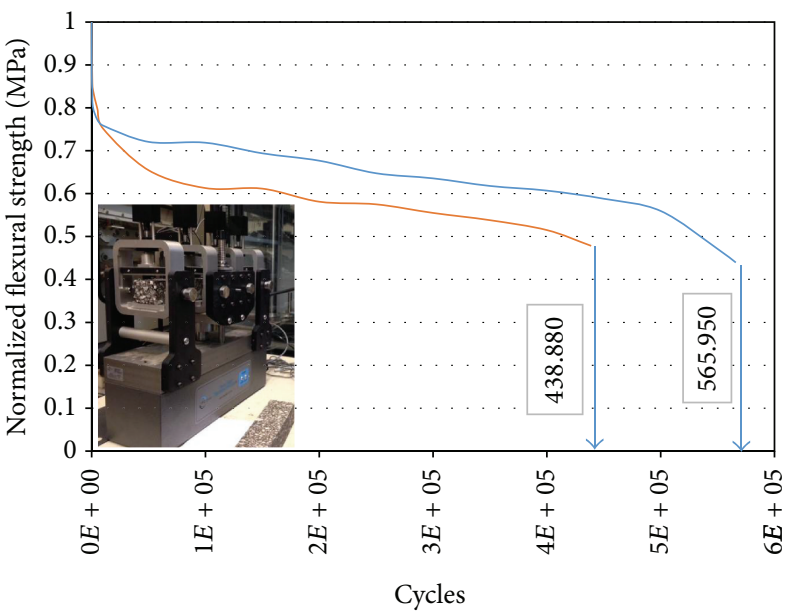

(a) STE-10

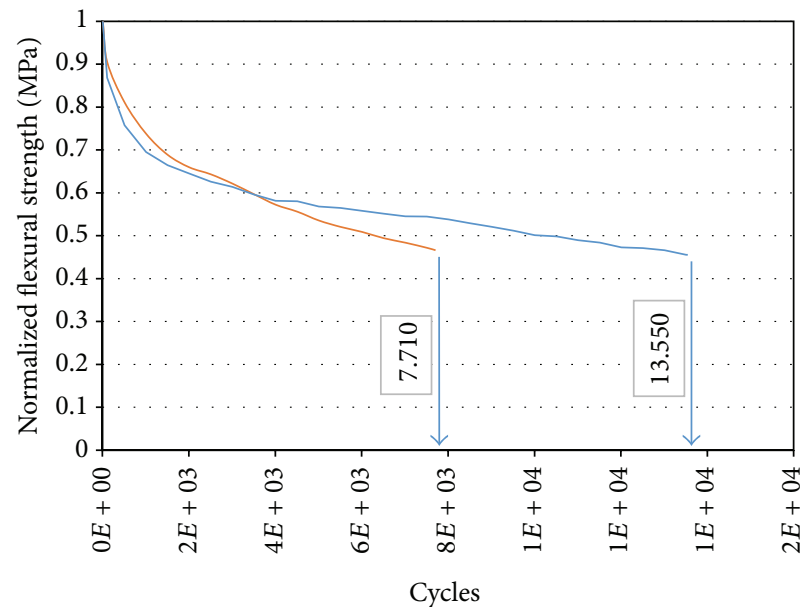

(b) ARMA

Figure 6: Beam fatigue test.

method, and permeability test. Only below aggregate gradation mix does not meet the design criteria of Marshall flow and VMA values at 3 percent air voids. The results indicate that the mix prepared by using upper aggregate gradation has the best performance on moisture susceptibility with the optimum asphalt content of 5.5 percent; however, the IDT strength of medium mixture is the highest among all. Further, the mixes of upper and medium gradation have lower value of permeable coefficient and stripping percentage. The authors suggest developing threshold value in image analysis method for different types of mixtures and conditions and learning more about the relationship between stripping percentage and IDT test results. Based on the test results and authors' experience, it is recommended to use aggregate gradations between medium and upper (fine graded aggregate) for ADFT system due to their appropriate moisture resistance and permeability. Also, the modified asphalt binder STE-10 which provides higher fatigue life and rutting life for the asphalt mixture is more suitable for ADFT system compared to the ARMA.

\section{Conflict of Interests}

The authors declare that there is no conflict of interests regarding the publication of this paper.

\section{Acknowledgment}

This research was supported by a grant from R\&D Program of the Korea Railroad Research Institute, Republic of Korea.

\section{References}

[1] J. G. Rose, P. F. Teixeira, and P. Veit, "International design practices, applications, and performances of asphalt /bituminous railway trackbeds," in GEORAIL 2011: Symposium international 
Géotechnique ferroviaire, Paris, France, 19-20 mai 2011, Volume $1,2011$.

[2] M. J. Hensley and J. G. Rose, "Design, construction and performance of hot mix asphalt for railway trackbeds," in Proceedings of the 1st World Conference of Asphalt Pavements, Sydney, Australia, 2000.

[3] M. Fang, Y. Qiu, J. G. Rose, R. C. West, and C. Ai, "Comparative analysis on dynamic behavior of two HMA railway substructures," Journal of Modern Transportation, vol. 19, no. 1, pp. 2634, 2011.

[4] E. R. Brown, P. S. Khal, F. L. Roberts, Y. R. Kim, D. Y. Lee, and T. W. Kennedy, Hot Mix Asphalt Materials, Mixture Design, and Construction, NAPA Research and Education Foundation, Lanham, Md, USA, 3rd edition, 2009.

[5] J. G. Rose and R. S. Bryson, "Hot mix asphalt railway trackbeds: trackbed materials, performance evaluations, and significant implications," in Proceedings of the International Conference on Perpetual Pavements, Columbus, Ohio, USA, 2009.

[6] M. Fang, Y. Qiu, C. Ai, and Y. Wei, "Gradation determination of impermeable asphalt mix on subgrade surface layer for ballastless track in high-speed railway lines," in Proceedings of the 3rd International Conference on Transportation Engineering (ICTE '11), pp. 1926-1931, July 2011.

[7] J. G. Rose, P. F. Teixeira, and N. E. Ridgway, "Utilization of asphalt/bituminous layers and coatings in railway trackbeds: a compendium of international applications," in Proceedings of the ASME Joint Rail Conference (JRC '10), pp. 239-255, Urbana, Ill, USA, April 2010.

[8] E. Sangsefidi, H. Ziari, and A. Mansourkhaki, "The effect of aggregate gradation on creep and moisture susceptibility performance of warm mix asphalt," International Journal of Pavement Engineering, vol. 15, no. 2, pp. 133-141, 2014.

[9] F. Merusi, A. Caruso, R. Roncella, and F. Giuliani, "Moisture susceptibility and stripping resistance of asphalt mixtures modified with different synthetic waxes," Journal of the Transportation Research Board, no. 2180, pp. 110-120, 2010.

[10] European Asphalt Pavement Association (EAPA), Asphalt in Railway Tracks, European Asphalt Pavement Association, Breukelen, The Netherlands, 2010.

[11] AASHTO, "Specific gravity and absorption of coarse aggregate," AASHTO T 85-10, 2010.

[12] AASHTO, "Specific gravity and absorption of fine aggregate," Tech. Rep. T 84-10, American Association of State Highway and Transportation Officials, 2010.

[13] AASHTO, "Standard method of test for resistance of compacted asphalt mixtures to moisture-induced damage," AASHTO T 283-03, 2003.

[14] Matlab, “The MathWorks,” 2013, http://www.mathworks.com/.

[15] Florida Department of Transportation (FDOT), "Measurement of water permeability of compacted asphalt paving mixtures," Tech. Rep. FM 5-565, Florida Department of Transportation, Tallahassee, Fla, USA, 2000.

[16] S. H. Lee, J. W. Lee, D. W. Park, and H. V. Vo, "Evaluation of asphalt concrete mixtures for railway track," Construction and Building Materials, vol. 73, pp. 13-18, 2014.

[17] R. Dongré, J. D’Angelo, and A. Copeland, "Refinement of flow number as determined by asphalt mixture performance tester: use in routine quality control-quality assurance practice," Transportation Research Record, no. 2127, pp. 127-136, 2009.

[18] AASHTO, "Standard method of test for determining the fatigue life of compacted hot-mix asphalt (HMA) subjected to repeated flexural bending," Tech. Rep. AASHTO T 321-03, 2003. 

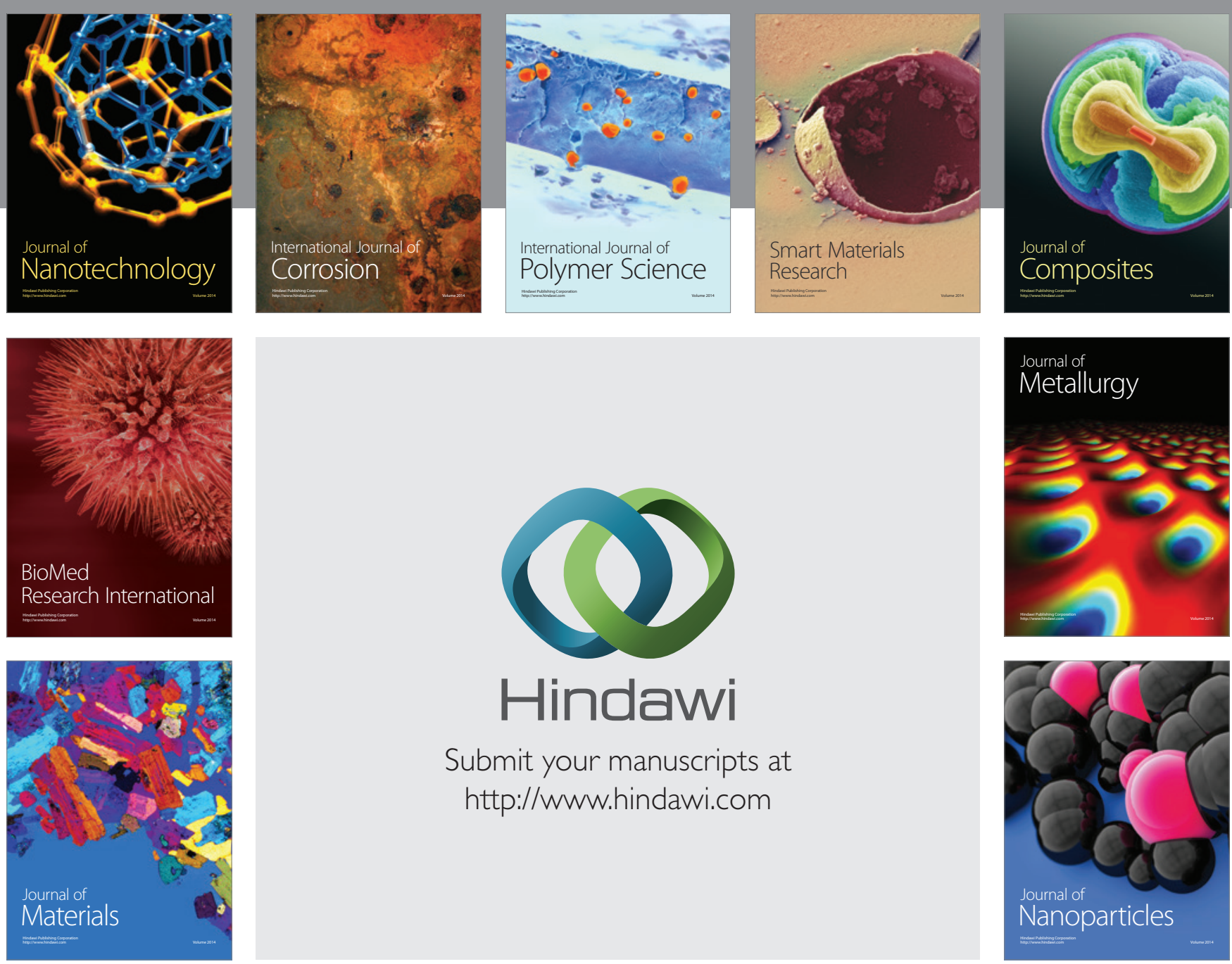

Submit your manuscripts at http://www.hindawi.com
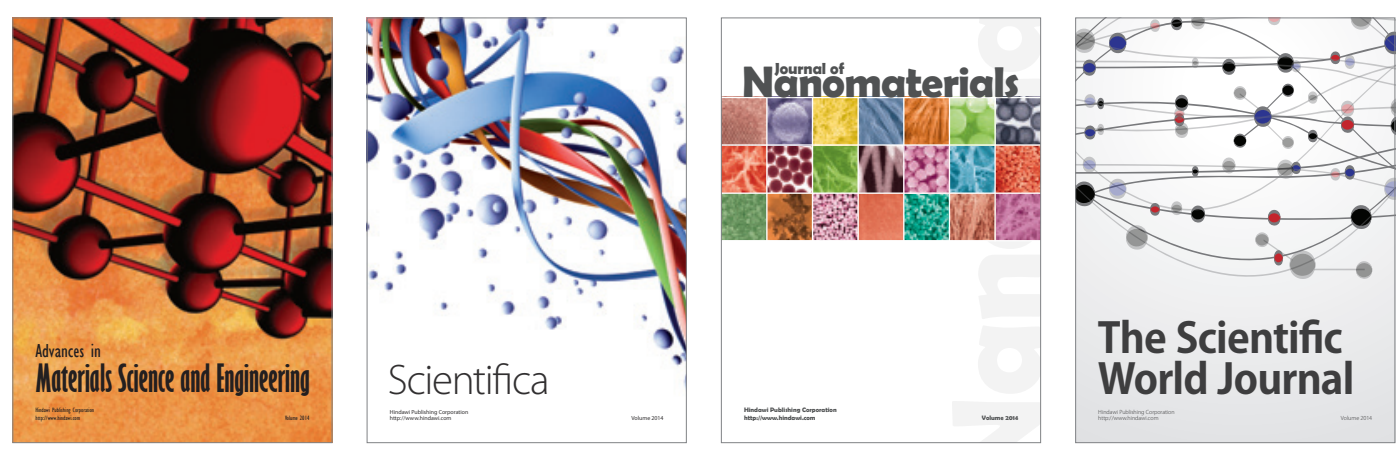

\section{The Scientific World Journal}
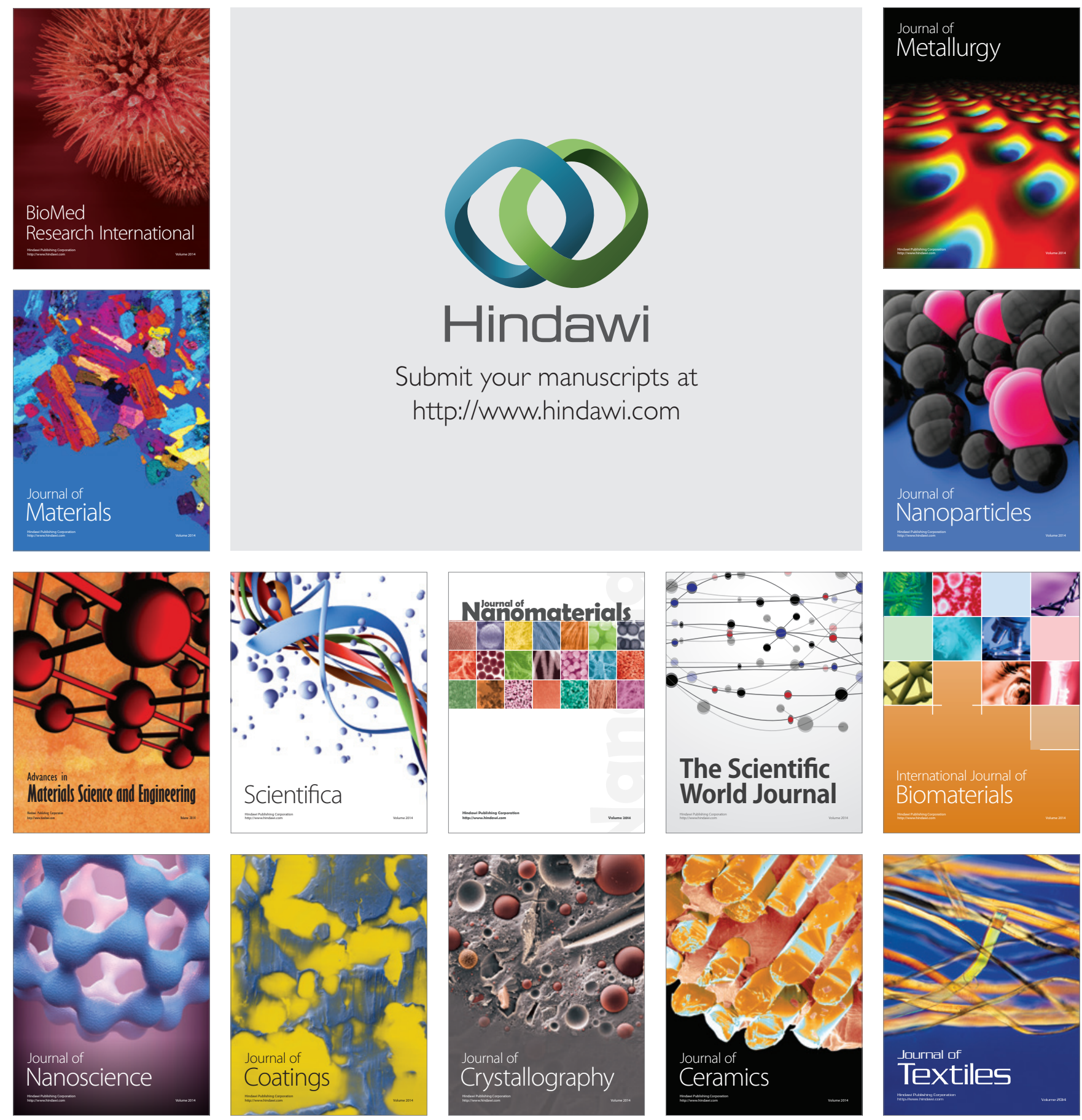